\title{
A Darwinian approach to the origin of life cycles with group properties
}

Armin Rashidi ${ }^{1 *}$, Deborah E. Shelton ${ }^{1}$, Richard E. Michod ${ }^{1}$

${ }^{1}$ Department of Ecology and Evolutionary Biology, University of Arizona, Tucson, AZ 85721

Corresponding author:

Email: arminrashiditucson@gmail.com (AR)

Keywords: multicellularity; fitness; palintomy; growth; mortality

Short title: The origin of group cycles 


\section{Abstract}

A selective explanation for the evolution of multicellular organisms from unicellular ones requires knowledge of both selective pressures and factors affecting the response to selection. Understanding the response to selection is particularly challenging in the case of evolutionary transitions in individuality, because these transitions involve a shift in the very units of selection. We develop a conceptual framework in which three fundamental processes (growth, division, and splitting) are the scaffold for unicellular and multicellular life cycles alike. We (i) enumerate the possible ways in which these processes can be linked to create more complex life cycles, (ii) introduce three genes based on the growth, division and splitting that, acting in concert, determine the architecture of the life cycles, and finally, (iii) study the evolution of the simplest five life cycles using a heuristic model of coupled ordinary differential equations in which mutations are allowed in the three genes. We demonstrate how changes in the regulation of three fundamental aspects of colonial form (cell size, colony size, and colony cell number) could lead unicellular life cycles to evolve into primitive multicellular life cycles with group properties. One interesting prediction of the model is that selection generally favors cycles with group level properties when intermediate body size is associated with lowest mortality. That is, a universal requirement for the evolution of group cycles in the model is that the size-mortality curve be Ushaped. Furthermore, growth must decelerate with size.

\section{Author Summary}

Factors influencing the early evolution of life cycles with group properties are largely undefined. A theoretical framework is provided here in which three fundamental processes (growth, division, and splitting) shape the architecture of the simplest five life cycles and their evolution. We demonstrate the critical importance of three fundamental aspects of colonial form (cell size, colony size, and colony cell number) in the transition from unicellular life to primitive multicellular life cycles. Our model reveals selection pressure for cycles with group properties when intermediate body size is associated with lowest mortality (i.e. U-shaped size-mortality relationship) and growth decelerates with colony size. This heuristic model provides a conceptual framework which could be used to further study the evolution of earliest colonial life cycles. 


\section{Introduction}

"And what is it, then, which is immortal? Clearly not the substance, but only a definite form of activity...the cycle of material which constitutes life returns even to the same point and can always begin anew, so long as the necessary external conditions are forthcoming...the cycle of life, i.e. of division, growth by assimilation and repeated division, should [n]ever end; and this characteristic it is which I have termed immortality. It is the only true immortality to be found in Nature-a pure biological conception, and one to be carefully distinguished from the eternity of dead, that is to say, unorganized, matter." Weismann (1890)

A life cycle consists of successive states through which life passes until it returns to some initial state. Division, growth, and splitting are the minimal constituents of a life cycle, and the way these three processes are organized in time and space defines the broad outline of development-defined by Griesemer [1] to be the acquisition of the capacity to reproduce. Division is the simplest form of reproduction, without which the life cycle will eventually go extinct as some mortality is unavoidable. It necessitates the production of new material, i.e. growth. Finally, some kind of a splitting process, by which the products of reproduction go their own ways and complete the cycle, is required. An important question concerns the way these three basic processes at the cellular level are assembled to produce more complex forms during the initial stages of the evolutionary transition from unicellular to multicellular life.

There is a brief multicellular state before splitting inherent in all unicellular life cycles when daughter cells have been produced by cell division and are still held together. This state is transient and represents an inevitable state through which the unicellular life cycle must pass. In most multicellular forms of life, the single-celled state tends to be transient and often restricted to single-celled propagules or gametes. Intermediate levels of complexity, by which we mean a significant contribution to the life cycle of both single-celled and colonial states, are the focus here. Such mixed life cycles must have been represented in transitional forms if the evolutionary transition to multicellularity involved small continuous steps as Darwin proposed in his explanation of the evolution of complex organs such as the human eye.

We investigate how and why simple unicellular life cycles become more complex and begin to involve colonial states and processes that operate at the cell group (i.e. multicellular) level. We 
investigate the conditions under which small modifications (resulting from random mutations) of unicellular life cycles could lead to the evolution of stable, more complex cycles that represent the presumed transitional forms on the way to multicellular life.

\section{Materials and Methods}

\section{States, transitions, and mortality}

For the purpose of analytical tractability of the explicit mathematical model given below, we impose an upper limit of four-fold growth of a single cell yielding, with two divisions, four daughter cells. We consider single cells, 2-celled colonies, and 4-celled colonies and define body size (unicellular or multicellular) as total cellular volume. Since we are studying the earliest stages of evolution of colonial life cycles, we ignore the contribution of the extracellular matrix to body size. We measure size relative to the size of a single small cell given as $\mathrm{x}$. We include single cells with three different sizes $(1 \mathrm{x}, 2 \mathrm{x}$, and $4 \mathrm{x}), 2$-celled colonies in which the colonies have two different sizes ( $2 x$ and $4 x$ ), and 4-celled colonies composed of $1 x$-sized cells (4x). A 2x cell and 2-celled colonies composed of $1 \mathrm{x}$ cells have the same body size in our model as they have the same total cellular volume. Likewise, a single $4 \mathrm{x}$ cell, 2-celled colonies composed of $2 \mathrm{x}$ cells, and the 4-celled colony composed of $1 \mathrm{x}$ cells are also assumed to have the same body size. We have a total of 6 states in the model, each defined by body size and the number of cells it contains (Figure 1). We denote a state by the general notation $\mathrm{N} x \mathrm{~S} y$, where $\mathrm{N}$ represents Number with $x$ being the number of cells in the state $(1=$ single cell, $2=2$-celled colony, and $4=4$-celled

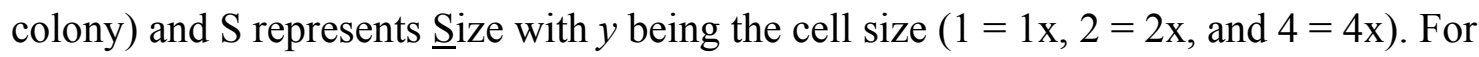
example, $\mathrm{N} 2 \mathrm{~S} 2$ represents a 2-celled colony consisting of cells with size 2x.

During its life cycle, the organism transitions from one state to the next. We consider all possible transitions between states (Figure 2). The parameter associated with each arrow in Figure 2 shows the time required for the corresponding transition to take place. The three transitions that constitute a life cycle are: $(i)$ growth, a cell or colony (uni- or multicellular) grows 2-fold in size without increasing the number of cells; (ii) division (asexual), one or two rounds of mitosis during which a single cell becomes a 2-celled (one round of mitosis) or a 4-celled (two rounds of 
mitosis) colony, or a 2-celled colony becomes a 4-celled colony; and (iii) splitting, the cells comprising the colony split from the colony and go their own way. We denote the transition times for growth, division, and splitting by $g, d$, and $s$, respectively. $g 1$ and $g 2$ are the transition times for the growth of N1S1 to N1S2 and N1S4, respectively. As discussed below, we ignore cooperation and other kinds of interactions among cells within groups. Consequently, we assume that being in the colony per se does not affect the rate of growth. Specifically, a 1x-sized cell is assumed to have the same growth rate whether it grows as an independent single cell ( $g 1)$ or within a colony $(g 3)$. This means $g 3=g 1$. We assume that division and splitting are significantly more rapid transitions than growth, thus $d=s=0$. This is consistent with the fact that mitosis typically takes up only a small portion of the life cycle [2], while growth takes longer as it requires production of new biomaterial. A group property in our model is represented by a transition that begins with and ends in a group state (red arrows in Figures 1 and 2). There are two such transitions in the model: transition from N2S1 to N2S2 (growth as a group) and from $\mathrm{N} 2 \mathrm{~S} 2$ to N4S1 (division within the group). Transitions from a group state to a non-group state (e.g. N2S1 to N1S1) do not represent a group property because the presence of group states such as $\mathrm{N} 2 \mathrm{~S} 1$ is required to form a cycle.

Mortality $(\mu)$ is the probability of dying in a certain state before making a transition to another state and is assumed to depend strictly on body size. Hence, states with the same size are assumed to have equal mortality values. $\mu 1, \mu 2$, and $\mu 4$ denote the probabilities of death in $1 \mathrm{x}$, $2 \mathrm{x}$, and $4 \mathrm{x}$ states, respectively.

\section{Genotypes}

Each cycle is encoded in the model by an asexual haploid genotype. At least three genes are required to join together the basic life cycle properties of growth, division and splitting. While there are a variety of choices, the genes we have chosen are motivated by evidence involving cell cycle control in simple colonial organisms (see Discussion): c (maximum cell size), $C$ (maximum colony size), and $N$ (maximum colony cell number). Each gene has two possible alleles, allele 2 or 4 . The cell can reach, outside of a colony, a size of $2 \mathrm{x}$ when $c=2$ and $4 \mathrm{x}$ when $c=4$. Similarly, the colony can reach a size of $2 \mathrm{x}$ when $C=2$ and $4 \mathrm{x}$ when $C=4$. The colony 
can have a maximum of 2 cells when $N=2$ and 4 cells when $N=4$; it cannot hold together more cells than determined by gene $N$. We identify a genotype by the triplet $(c, C, N)$.

\section{Cycles}

Genotypes determine life cycles. We impose the following rules for genotype-phenotype mapping: (i) Growth supersedes other transitions. When the organism has a choice to grow or undergo another transition, it grows; (ii) colonies do not split to colonies; they split to cells; and (iii) once division has started, it continues without a pause as long as the genotype allows. It will stop only when further division would contradict the gene $N$ or the cells become smaller than 1x.

We define a minimal cycle as one that cannot be further reduced. In other words, no shortcut exists between any two states of a minimal cycle. For example, N1S1-N1S2-N1S4-N4S1-N2S1N1S1 is not a minimal cycle because it can be reduced to N1S1-N1S2-N2S1-N1S1 by a shortcut between states N1S2 and N2S1. A minimal cycle is always fitter than a non-minimal one because the non-minimal cycle involves additional states that not only retard the rate of cycling but also add to the mortality risk. Since non-minimal cycles are always dominated by their minimal counterparts, we do not consider non-minimal cycles in our analysis. The following five minimal life cycles can be identified in Figure 1: N1S1-N1S2-N2S1-N1S1 (cycle 1 or small fission), N1S2-N1S4-N2S2-N1S2 (cycle 2 or large fission), N1S1-N1S2-N1S4-N4S1-N1S1 (cycle 3 or palintomy), N1S2-N2S1-N2S2-N1S2 (cycle 4 or reverse fission), and N1S1-N1S2N2S1-N2S2-N4S1-N1S1 (cycle 5 or the complex cycle). Important features of each cycle and the genotype associated with it are detailed in Table 1 . As an example, genotype $(2,4,4)$ determines cycle 5 by the following mechanism: N1S1 grows 2-fold and transitions to N1S2. Since N1S2 cannot grow any further due to $c=2$, it divides and produces N2S1. N2S1 cannot divide again because its cells are already 1x. So, it grows by default and produces N2S2. N2S2 is a $4 \mathrm{x}$-sized colony and cannot grow further because $C=4$. So, it divides and produces N4S1. N4S1 cannot divide again because its cells are already 1x. So, it splits into 1x cells and the cycle is completed. The interested reader may go through other cycles and genotypes in a similar way. 
With one exception, each cycle is produced by a unique genotype. The exception concerns cycle 1 , which can be produced by either of the two genotypes $(2,2,2)$ and $(2,2,4)$. This is because when $C=2$, that is, the colony can reach a size of $2 x$ at most, the colony can have a maximum of 2 cells regardless of the whether $N=2$ or 4 . In other words, the potential offered by $N=4$ of having 4 cells in the colony cannot realize when $C=2$. Since we will be considering mutations that convert genotypes to one another (see below), we define a virtual cycle, called cycle 1', which is essentially the same as cycle 1 but associated with genotype $(2,2,4)$. The purpose of doing this becomes clearer in the next section. Finally, genotypes $(4,2,2)$ and $(4,2,4)$ do not produce a phenotype because $c=4$ would result in a $4 \mathrm{x}$ cell, which is a dead end given $C=2$. Figure 2 shows the cycles with their associated genotypes.

\section{Mutations}

Mutations in the model occur randomly and change the value of a gene from 1 to 2 or vice versa. Two categories of mutations can be identified. $(i)$ mutations that convert one genotype to another genotype which is associated with another cycle. We call this class "between-mutations" because they correspond to going from one cycle to another cycle. The $(4,4,4)$-to- $(2,4,4)$ mutation, which converts cycle 3 to cycle 5, is an example for this class. The only exception is the mutations between cycles 1 and $1^{\prime}$, which in principle do not convert cycles. (ii) mutations that convert a genotype to another genotype that is a dead end. The $(4,4,4)$-to- $(4,2,4)$ mutation is an example for this class. For simplicity of analysis, we assume all mutations have the same rate. Embedded in Figure 2 is the mutation diagram. All mutations are bidirectional. Cycle 1 can mutate to cycles $1^{\prime}$ or 4 , cycle $1^{\prime}$ to cycles 1 or 5 , cycle 2 to cycles 3 or 4 , cycle 3 to cycles 2 or 5 , cycle 4 to cycles 2 or 5 , and finally cycle 5 to cycles $1^{\prime}, 3$, or 4 .

As an example, the $(2,4,2)$ genotype defines cycle 4 . The $2 x$ cell in this cycle has to divide because $c=2$ allows for a maximum size of $2 \mathrm{x}$ for a cell outside the colony. The 2x 2-celled colony then grows to form the $4 \mathrm{x} 2$-celled colony, which has to spilt because $C=4$ and $N=2$ allow for a maximum size of $4 \mathrm{x}$ and maximum cell number of 2 for a colony, respectively. A mutation in gene $c$ from 1 to 2 enables the cell to grow to the size $4 \mathrm{x}$ before entering mitosis. The 
4x cell divides and forms the $4 \mathrm{x} 2$-celled colony which has to split because it is at the largest size allowed by $C=4$. Therefore, mutation from $(2,4,2)$ to $(4,4,2)$ converts cycle 4 to cycle 2 .

\section{Model}

The generic equation for the dynamics of a life cycle in our model is

$\frac{d x_{i}}{d t}=$ self rep + Between $_{+}\left(x_{i}\right)-$ Between $_{-}\left(x_{i}\right)-$ Out $\left(x_{i}\right)-\operatorname{adj} u s t_{i}$,

where self repis the rate of growth for cycle $i$ due to self-replication (in the absence of mutations), Between $n_{+}\left(x_{i}\right)$ is the rate of other cycles turning into cycle $i$ as a result of betweenmutations, Between- $\left(x_{i}\right)$ is the rate by which cycle $i$ turns into other cycles as a result of between-mutations, $\operatorname{Out}\left(x_{i}\right)$ is the rate by which the genotype of cycle $i$ turns into a dead end, and adj $u s t_{i}$ is the term included to keep the population size constant and is defined by

$\operatorname{adj} u s t=\frac{-x_{i}}{\sum_{i} x_{i}}\left[\sum_{i}\left(\right.\right.$ self rep - Out $\left.\left.\left(x_{i}\right)\right)\right]$.

Let us consider cycle 1 by assuming that $x_{1}(0)$ cells start the cycle. The time required for each round is $g 1$. Ignoring mutations for the time being, the expected number of offspring that are produced by the cycle in the first round is $2 x_{1}(0)(1-\mu 1)(1-\mu 2)^{2}$, taking account of the risks of mortality in each state and the fact that splitting from N2S1 to N1S1 produces two offspring. Also note that the time required for division and splitting is negligible by assumption. Since each offspring carries the same genotype as the parent, it will be counted as one organism following cycle 1. Using $t$ to denote time, the dynamics of cycle 1 can then be described as $x_{1}(t)=x_{1}(0)\left[2(1-\mu 1)(1-\mu 2)^{2}\right]^{\frac{t}{g 1}}$, or by the differential equation

self rep $=\frac{x_{1} \operatorname{Ln}\left[2(1-\mu 1)(1-\mu 2)^{2}\right]}{g 1}$.

Bringing mutations into consideration, we have

$$
\begin{aligned}
& \text { Between }_{+}\left(x_{1}\right)=\sigma\left(x_{1^{\prime}}+x_{4}\right) \\
& \text { Between_}_{-}\left(x_{1}\right)=2 \sigma x_{1} \\
& \text { Out }\left(x_{1}\right)=\sigma x_{1} .
\end{aligned}
$$


Note that cycles 1 ' and 4 are the only cycles converted to cycle 1 by a single between-mutation. Similarly, cycle 1 is converted to cycles 1' and 4 by a single between-mutation (for each conversion). Finally, there is one out-mutation, from $(2,2,2)$ to $(4,2,2)$, that converts the genotype of cycle 1 to a dead end. Substituting (3) and $(4.1,4.2,4.3)$ in (1) yields

$\frac{d x_{1}}{d t}=\frac{x_{1} \operatorname{Ln}\left[2(1-\mu 1)(1-\mu 2)^{2}\right]}{g 1}+\sigma\left(x_{1^{\prime}}+x_{4}\right)-2 \sigma x_{1}-\sigma x_{1}-\operatorname{adj} u s t_{1}$,

where adj ust is defined using (2).

As another example, consider cycle $3 . x_{3}(0)$ cells start the cycle, and the time required for each round is $g 1+g 2$. Ignoring mutations for the time being, the expected number of offspring that are produced by the cycle in the first round is $4 x_{3}(0)(1-\mu 1)(1-\mu 2)(1-\mu 4)^{2}$, considering that splitting from N4S1 to N1S1 produces four offspring. The dynamics of cycle 3 can then be described as $x_{3}(t)=x_{3}(0)\left[4(1-\mu 1)(1-\mu 2)(1-\mu 4)^{2}\right]^{\frac{t}{g 1+g 2}}$, or by the differential equation self rep $=\frac{x_{3} \operatorname{Ln}\left[4(1-\mu 1)(1-\mu 2)(1-\mu 4)^{2}\right]}{g 1+g 2}$.

Bringing mutations into consideration, we have

$$
\begin{aligned}
& \text { Between }_{+}\left(x_{3}\right)=\sigma\left(x_{2}+x_{5}\right) \\
& \text { Between }_{-}\left(x_{3}\right)=2 \sigma x_{3} \\
& \operatorname{Out}\left(x_{3}\right)=\sigma x_{3} .
\end{aligned}
$$

Note that cycles 2 and 5 are the only cycles converted to cycle 3 by a single between-mutation. Similarly, cycle 3 is converted to cycles 2 and 5 by a single between-mutation (for each conversion). Finally, there is one out-mutation, from $(4,4,4)$ to $(4,2,4)$, that converts the genotype of cycle 3 to a dead end. Using the same procedures as for cycle 1, we have

$$
\frac{d x_{3}}{d t}=\frac{x_{3} \operatorname{Ln}\left[4(1-\mu 1)(1-\mu 2)(1-\mu 4)^{2}\right]}{g 1+g 2}+\sigma\left(x_{2}+x_{5}\right)-2 \sigma x_{3}-\sigma x_{3}-\text { adj ust. }
$$

The same method is applied to derive equations for the other 4 cycles: 


$$
\begin{aligned}
& \frac{d x_{1^{\prime}}}{d t}=\frac{x_{1^{\prime}} \operatorname{Ln}\left[2(1-\mu 1)(1-\mu 2)^{2}\right]}{g 1}+\sigma\left(x_{1^{\prime}}+x_{5}\right)-2 \sigma x_{1^{\prime}}-\sigma x_{1^{\prime}}-\text { adj ust. } \\
& \frac{d x_{2}}{d t}=\frac{x_{2} \operatorname{Ln}\left[2(1-\mu 2)(1-\mu 4)^{2}\right]}{g 2}+\sigma\left(x_{3}+x_{4}\right)-2 \sigma x_{2}-\sigma x_{2}-\text { adj ust } \\
& \frac{d x_{4}}{d t}=\frac{x_{4} \operatorname{Ln}\left[2(1-\mu 2)^{2}(1-\mu 4)\right]}{g 1}+\sigma\left(x_{1}+x_{2}+x_{5}\right)-3 \sigma x_{4}-\text { adj ust }_{4} \\
& \frac{d x_{5}}{d t}=\frac{x_{5} \operatorname{Ln}\left[4(1-\mu 1)(1-\mu 2)^{2}(1-\mu 4)^{2}\right]}{2 g 1}+\sigma\left(x_{1^{\prime}}+x_{3}+x_{4}\right)-3 \sigma x_{5}-\text { adjust. }
\end{aligned}
$$

It can be shown that $\sum_{i} \frac{d x_{i}}{d t}=1$. The adjustment term adds to or detracts from each cycle according to its relative frequency in the population, such that the population size remains constant at 1 all the time.

\section{Implementation}

We have 6 ordinary differential equations, 6 variables $\left(x_{1}, x_{1}, x_{2}, x_{3}, x_{4}, x_{5}\right)$ and 5 parameters ( $g 1, g 2, \mu 1, \mu 2$, and $\mu 4)$. A mutation rate of $\sigma=10^{-3}$ is chosen in our simulations. Higher or lower values of $\sigma$ do not affect the general pattern of our results. Since we are interested in the initial evolution and spread of group life cycles from rarity, we start from a population composed initially of only the simplest of cycles, that is, $x_{1}(0)=1$ and $x_{i}(0)=0$ for all $i \neq 1$. We have the following 12 non-overlapping regions (Figure 3): (region 1: $\mu 1>\mu 2>\mu 4$ and $g 1>g 2$ ), (region 2: $\mu 1>\mu 2>\mu 4$ and $g 2>g 1$ ), (region $3: \mu 1>\mu 4>\mu 2$ and $g 1>g 2$ ), (region $4: \mu 1>\mu 4>\mu 2$ and $g 2>g 1$ ), (region 5: $\mu 2>\mu 1>\mu 4$ and $g 1>g 2$ ), (region 6: $\mu 2>\mu 1>\mu 4$ and $g 2>g 1$ ), (region 7: $\mu 2>\mu 4>\mu 1$ and $g 1>g 2),($ region 8: $\mu 2>\mu 4>\mu 1$ and $g 2>g 1),($ region 9: $\mu 4>\mu 2>\mu 1$ and $g 1$ $>g 2$ ), (region 10: $\mu 4>\mu 2>\mu 1$ and $g 2>g 1$ ), (region 11: $\mu 4>\mu 1>\mu 2$ and $g 1>g 2$ ), and (region 12: $\mu 4>\mu 1>\mu 2$ and $g 2>g 1)$. We are interested to see which cycle(s) become dominant or extinct in each region. In each region, we choose 100 random points using Python 2.5.2, and simulate the system. Each point has five coordinates corresponding to the five parameters. Simulations are continued until all cycles reach a steady state. To make sure that the population does not go extinct altogether, we did each simulation first without the adj ust terms, and 
proceeded with simulating the full system only if at least one cycle continued to grow exponentially. Simulations are performed in Mathcad 14 and the code is available upon request.

\section{Results}

Figure 3 shows, in a schematic way, the cycles that evolved in each region and Figure 4 shows typical examples in different regions and their boundaries. In all regions, all cycles reached a steady state and no persistent oscillatory behavior was observed. When a cycle moved from their initial frequency, one of the three patterns was observed: ( $i$ ) steady rise to a steady state frequency, (ii) steady decline to a steady state frequency, or (iii) initial rise to a maximum followed by a decline to zero. Condition (iii) corresponds to cases where a cycle served as a transition platform for another cycle to reach dominance. Except on the boundaries between regions 3 and 11 (Figures 3 and 4H) and between regions 4 and 12 (Figures 3 and 4J), where three cycles coexisted at a significant frequency, there was one dominant cycle that reached a frequency of 1 in each region. Region 3 was the only exception in which 2 cycles (palintomy and reverse fission) evolved. In the following discussion, we put cycles 1 and 1' in one group and call it cycle 1. Likewise in all diagrams, the frequency of cycle 1 is in fact the sum of the frequencies of cycle 1 and cycle $1^{\prime}$.

Cycle 3 (palintomy) was the most successful cycle, the only cycle that evolved in regions 5-8 and the majority of regions 2, 3, 9, and 10. Cycle 3 also extended into the boundary areas of regions 1,11 , and 12 . Cycle 1 was the second most successful cycle, dominating the majority of regions 11 and 12, and extending into the boundary areas of regions 3, 4, 9, and 10. Cycle 4 dominated the majority of region 4 and extended into the boundary areas of regions $2,3,11$, and 12. It also evolved in a small part within region 3. Cycle 2 evolved exclusively within region 1. Finally, cycle 5 was the least successful cycle, evolving only in a small boundary area between regions 4 and 12 .

\section{Discussion}

\section{General conclusions from the results}


The transition from life as a single cell to life as a multicellular organism has occurred many times and it has led to several important evolutionary innovations such as large body size, cellular differentiation and division of labor $[3,4]$. Our focus here is on conditions that promote the evolution of cycles with group properties. Growth as a group occurs when the cells grow within a colony. Division within the group occurs when cells divide while staying in the mother colony. Cycle 4 exhibits growth as a group when transitioning from N2S1 to N2S2. Cycle 5, on the other hand, exhibits both growth as a group (when transitioning from N2S1 to N2S2) and division within a group (when transitioning from N2S2 to N4S1). Generally speaking, moving from left to right in Figure 3, group cycles start to evolve. Regions 5-10 and all their boundaries are completely dominated by non-group cycles. The common feature of these regions is $\mu 2>\mu 1$. Region 1 is also completely dominated by a non-group cycle. These observations suggest that for the most part, natural selection in the model favors cycles without group properties. Interestingly, the evolutionary pathways from cycle 1 to cycle 2 or 3 have to pass through at least one of the cycles with group properties (cycles 4 and 5). When evolution favors non-group cycles, the group cycles serve only as transitional steps and do not spread enough to reach a significant frequency in the population.

Of the two group cycles, cycle 4 did far better, almost completely dominating region 4 and also a small part of region 3. Cycle 5, on the other hand, only evolved to a significant frequency in a small part of the boundary between regions 4 and 12. Coexistence of three cycles was an interesting observation which occurred on the boundaries between regions 3 and 11, and also between regions 4 and 12. On the former, an increase in small- and large-size mortality (i.e. $\mu 1$ and $\mu 4$, respectively) promotes the evolution and spread of a group cycle, namely cycle 4 (Figure $4 \mathrm{H})$. On the latter, a decrease in small- and large-size mortality (i.e. $\mu 1$ and $\mu 4$, respectively) prevents the extinction of a group cycle, namely cycle 5 (Figure $4 \mathrm{~J}$ ). The boundary zone between regions 4 and 12 is the only place where both group cycles can evolve. The universal and the most important requirement for the evolution of group cycles was the association between the intermediate size $(2 \mathrm{x})$ and lowest mortality. This is consistent with common sense because cycles 4 and 5 have the largest proportion of their cycles in the intermediate size states $(2 / 3$ and 2/5 of cycles 4 and 5, respectively). The relationship between size and mortality, however, was a 
necessary but not sufficient condition. While deceleration of growth with size $(g 2>g 1)$ was a requirement for the evolution of cycle 5, cycle 4 had a chance to evolve regardless of the association between size and growth rate (Figure 4H and 4J). Deceleration of growth with size disfavors the cycles with the $g 2$ transition, including cycles 2 and 3.

In summary, the evolution of group division (i.e. cycle 5) in the model requires growth deceleration with size and low mortality values with a symmetric U-shaped size-mortality curve (with the lowest mortality being associated with the intermediate size). We call this condition

(a). Growth within the group can evolve when the intermediate size confers the lowest mortality and one of the following conditions is met: $(i)$ condition $(a)$ as a property of cycle 5 , (ii) growth deceleration with size and $\mu 1>\mu 4$ (region 4), or (iii) growth acceleration with size, $\mu 1>\mu 4$, and small $\mu 2$ (small area in region 3). Conditions (ii) and (iii) promote the evolution of growth within the group as a property of cycle 4 .

\section{Size-mortality and size-growth relationships}

Size-mortality relationships are complex. Large sizes in phytoplankton have been shown to afford protection against zooplankton grazing [5], photoinhibition [6], and viral infection [7]. Similarly, multicellular colonies of the green alga, Chlorella vulgaris, enjoy a fitness advantage (immunity against predation) due to their relatively large size [8]. During the initial evolution of groups in the volvocine green algae, lower mortality in the group state (due to a release from predation) seems to have been the driving force for the smallest sized colonies consisting of 4-8 cells [9]. Recent experiments have shown than Chlamydomonas cells form facultative groups of 10-100,000 cells in the presence of predators. When the predator is removed, Chlamydomonas reverts back to unicellular life cycles [10]. Marbà et al [11] demonstrated an inverse relationship between size and mortality across plant species. There may, however, be disadvantages with large size. For example, one major disadvantage of large size in phytoplankton is high sinking velocity [12]. Refuge from predation offered by small size has been described in very small metazoans and phytoplankton [13-15]. Trait compensation is another example. Large size in snails reduces morphological vulnerability to the shell-entry predation tactics used by crayfish. 
However, small prey shows stronger antipredator behavior than large prey (i.e. behavioral compensation for morphological vulnerability) [16].

Our results regarding size-growth relationships suggest that while the relationship between mortality and body size has been an important determinant of selection for life cycles with group properties, it has not been the only determinant. Just like size-mortality relationships, the relationship between growth rate and size is not trivial [17]. A need to maximize the surface to volume ratio [18], which is usually advantageous, may pose certain constraints on growth rates of single cells of different size [19]. If diffusion of nutrients within the colony becomes an issue for growth of cells, colonial growth may also be constrained [20]. The growth of Amoeba proteus, for example, is fast initially and approaches zero towards the end of the life cycle [21]. This pattern, however, is not universal. The myxomycete Physarum polycephalum, for example, exhibits two peaks in macromolecular synthesis [22]. The growth rate of diatoms and dinoflagellates has weak dependence on cell volume or mass [23]. A recent review concluded that there is no universal pattern of growth in lower eukaryotes [24].

During the unicell-to-multicell evolutionary transition in individuality (ETI), the context of cell growth, division, and splitting changed drastically. In this model, which considers only the simplest life cycles, cell growth in the group context occurs in only two cycles and cell division in the group context occurs in only one cycle. Thus, it is impossible to separate the effects of these "group properties" from the architecture of the cycle in which they occur. While this model does not tease out the effect of group properties per se, it does highlight the fact that (given our constraints on the sizes of cells and colonies considered) group properties must first appear in the context of specific kinds of cycles. That is, the architecture of the cycles that include the group property in our model is a key factor in the first steps of the emergence of group properties.

\section{Relationship to empirical systems and previous modeling}

The genes with functions related to $c$ and $N$ have been identified in some cases. The size-division relationship at the cell level (the function of gene $c$ ) has been studied extensively in Schizosaccharomyces pombe. For this species, cell size triggers entry into mitosis because the 
interaction of two molecules that are localized to different parts of the cell declines in larger cells, triggering changes in the expression of genes related to mitosis $[25,26]$. The function of the $N$ locus is regulation of the ability of cells to adhere to one another. Genes for cadherins (a family of trans-membrane proteins important for cell-cell adhesion in Metazoa) are present in the choanoflagellate Monosiga brevicollis, a unicellular organism that is not known to have cell-cell contacts [27]. In their recent experimental evolution study, Ratcliff et al [28] selected for multicellularity in the unicellular yeast $S$. cerevisiae and observed the evolution of a "postdivision adhesion" trait that promoted the formation of yeast colonies. This observation provides support for the occurrence of mutations in our proposed gene $N$ that would favor the evolution of colonial forms. The evidence for gene $C$ is less compelling at this time and requires further genetic studies on model organisms.

Although the goal of our model was mainly heuristic, our results may be applied directly to organisms which, due to certain constraints, meet these assumptions. Cycles 1-4 are known to occur in volvocine green algae. Palintomy which is common in the volvocine green algae was also the most successful cycle in our model. Note that our model did not include the cost of discarding the cell wall during division or the cost of dividing during daylight, both aspects of volvocine biology that are thought to favor palintomy over binary fission [29]. We are not, however, aware of any example in this group that follows cycle 5 . This is consistent with the model, as cycle 5 was the least successful cycle.

A previous modeling attempt [30] to study the evolution of colonial life cycles considered two Chlamydomonas and one Gonium sociale (also known as Tetrabaena socialis) life cycles in separate synchronous generations of constant duration. Organisms were assumed to be exposed to lethal accidents and a higher fitness was modeled as a reduced accident rate. Assuming that the G. sociale cycle had a size-related selective advantage over the Chlamydomonas cycle, the conditions under which G. sociale would replace Chlamydomonas were investigated. In order to keep the population at steady state, accident rates, reproductive rate, or the carrying capacity were readjusted every generation. The results of that study confirm common sense. For a sufficiently high accident rate difference between the two life cycles, the one with a lower 
accident rate replaces the other. Importantly, and in contrast to the only previous work [28], we did not assume a single mortality value for a whole life cycle; rather mortality was a statespecific property determined by size.

Tarnita et al. [31] modeled the very first stages of evolutionary construction by two operations: staying together (ST; offspring resulting from reproduction stay together within the colony) and coming together (CT; separate entities join and form new colonies). Two types of units were considered and explicitly modeled: Type A units stay together after reproduction, while type B units always separate. The authors show that ST and CT are very different processes, go through different evolutionary landscapes, and represent different routes for the evolution of complexity. As opposed to ST, which is a clonal process (in the absence of mutations or sex), entities with varying degrees of relatedness may form complexes via CT. As a result, $\mathrm{CT}$ generates a platform for genetic diversity, whereas this platform can be provided by ST only via mutations during the growth of the aggregate. Evolution of ST occurs through linear selection, whereas CT evolution resembles evolutionary game dynamics. Stable co-existence of the two types (A and B) is possible in CT but not in ST. An interesting prediction of this study was that different environments can facilitate CT or ST. In an aquatic environment, for example, currents would make it difficult for separate units to find each other and, therefore, CT would be inefficient. This prediction is consistent with the idea that ST is characteristic of multicellular forms of aquatic origin as opposed to the terrestrial lineages [32]. Mechanisms for the evolution of cooperation were also studied in this model. ST was found to be stable under defection only when the rate of a mutation that creates mutants able to break from the colony and revert to solitary life was less than a certain error threshold. On the other hand, a specific mechanism for the evolution of cooperation is needed in CT given that CT represents an evolutionary game. Finally, the authors proposed that ST is an essential mechanism for the formation of higher-level individuals. We included ST (N2S1 to N2S2), but not CT, in our model and demonstrated the role of the architecture of life cycles in driving the evolution of group properties. Finally, mutations causing cooperation and defection were not modeled in our study (see below). Therefore, as opposed to the study by Tarnita et al. [31], stability to defection could not be explored in our model. 
In another recent theoretical work focusing on early evolution of life cycles with group properties, the origin of group reproduction was modeled by considering ST during the life cycle (e.g. fraction of time spent growing in group before separating; a life cycle trait) and a cellular life history trait (e.g. growing time) [33]. It was shown that with ST, and under certain conditions, cellular life cycle is no longer optimal. As a result, cellular life cycle traits change to adapt to the transient group context with features such as group reproduction. With continuous coevolution of cell cycle traits with the life history traits, group life cycles can form and function as the earliest step towards multicellular individuality. The evolution of group properties may therefore precede division of labor and specialization [34]. Our results from the present work demonstrate that this process can be facilitated by the very architecture of early life cycles.

\section{Limitations}

The advantage of the current model is its simplicity, which facilitates the analysis and simulations. The costs for this simplicity are the issues we have left out of the model. For example, we only considered the simple scenario in which colonies have a maximum of 4 cells and a 4-celled colony does not grow and produce colonies with larger number of cells. The model may be expanded in a straightforward way to incorporate larger groups, however analysis would be difficult. To ensure a 1:1 mapping between genotype and phenotype, we imposed three rules on transitions. Rules $(i)$ and (ii) deserve special attention. The first rule (growth supersedes other transitions) ensures that cycle 1 remains distinct from cycles 2 and 3. This is because N1S2 would otherwise have a choice to grow (and become part of cycles 2 or 3 ) or divide (and become part of cycle 1). Similarly, this rule ensures that cycle 1 remains distinct from cycles 4 and 5. This is because N2S1 would otherwise have a choice to grow (and become part of cycles 4 or 5) or split (and become part of cycle 1). Therefore, the first rule is plausible with the observation that oscillations between different cycles are infrequent in a given strain. It also eliminates the need for more complex genotypes in the model. Nevertheless, one could develop alternative (and perhaps more complex) genotype-phenotype mappings including a role for stochasticity. The second rule (colonies split to cells, not colonies) eliminates the N4S1-N2S1 transition. This does not cause any problem related to N4S1-N2S1-N1S1 pathway because cycles including this 
pathway would always be dominated by those different only in having the bypass transition N4S1-N1S1 with the same transition rate while avoiding the additional mortality of the N2S1 state. However rule (ii) also eliminates a potentially relevant cycle: N2S1-N2S2-N4S1-N2S1. This would be an interesting and unique cycle in that it has no single-cell state and all three transitions represent group properties. However, this cycle would be coded by genotype $(4,4,4)$, which also codes for cycle 3. To avoid a more complex genotype model, we imposed rule (ii). Obviously, an interesting expansion of this model would be using a 4-gene genotype and omitting rule (ii). Chain splitting (contradicting this rule) is known to occur in some bacterial species (e.g. cyanobacteria).

We do not consider here other aspects of group membership that may affect the growth and mortality of the different states, such as the possibility for cooperation and division of labor when in the colonial states. Cooperation among cells in a group, perhaps involving swimming, may be an advantage of the group life state and lead to the emergence of colonial forms. Similarly, by excluding social interactions and cooperation among cells at the start of the ETI, the present model inherently assumes that cooperation is derived. However, there are several examples of cooperation among single cells (e.g. cooperative interactions between bacteria), suggesting that single cells could have cooperated with each other before more complex life cycles with group properties arose. We developed the present model in its simplest form to find minimal conditions involving mortality for the emergence of group properties and as a heuristic platform for more complex models that include cell-cell interactions such as cooperation.

\section{Conclusions}

Using a mathematical model, we investigated the earliest stages of the evolution of colonial life cycles. This is an important question in the evolution of multicellularity and one that is seldom addressed. We included all possible life cycles that contain colonies of up to 4x size. We conclude that differences in mortality alone can select for group-level properties in cycles that lack them. The model predicts that selection will generally favor cycles with group-level properties when intermediate body size is associated with lowest mortality (i.e. the size-mortality curve is U-shaped) and growth decelerates with size. Our results highlight the importance of the 
structure (possible states and transitions) of early life cycles in the emergence of group properties.

Growth and mortality often depend on the size of the organism. The underlying causes of this relationship are usually not known and the role of these fundamental size scaling constraints during the multicellularity ETI is also not well understood [15]. The selective effects of the sizedependence of growth and mortality can be in conflict, for example, when faster growth occurs in smaller cell sizes but smaller cell sizes have greater risk of predation. In this case, selective pressures based on size-dependent growth would favor a cell cycle in which a cell splits as soon as it is large enough whereas selective pressure from predation would favor continued growth in larger cells. A clear solution to obviate such a conflict would be for divided cells to remain together, reaping the growth rate benefits of lowered surface-to-volume ratios and the mortalityavoidance benefits of larger overall size. This model shows that a scenario close to this one (intermediate optimal body size and faster growth in smaller cells) is associated with the emergence of cycles that have group properties, a major step towards a new (multicellular) level of organization.

\section{Acknowledgements}

This work was supported by NASA award NNX13AH41G and by NSF award MCB-1412395 to REM.

\section{References}

[1] Griesemer J (2001) The units of evolutionary transition. Selection 1: 67-80.

[2] Alberts B, Bray D, Lewis J, Raff M, Roberts K, Watson JD (1994) Molecular Biology of the Cell ( $\left.3^{\text {rd }} \mathrm{edn}\right)$. Garland Science. New York, USA.

[3] Kirk DL (2005) A twelve-step program for evolving multicellularity and a division of labor. Bioessays 27: 299-310.

[4] Herron MD, Hackett JD, Aylward FO, Michod RE (2009) Triassic origin and early radiation of multicellular volvocine algae. Proc Natl Acad Sci USA 106: 3254-3258.

[5] Lehman JT, Sandgren CD (1985) Species-specific rates of growth and grazing loss among 
freshwater algae. Limnol Oceanogr 30: 34-46.

[6] Visser PM, Passarge J, Mur LR (1997) Modelling vertical migration of the cyanobacterium Microcystis. Hydrobiologia 349: 99-109.

[7] Jacobsen A, Larsen A, Martinez-Martinez Z, Verity PG, Frischer ME (2007) Susceptibility of colonies and colonial cells of Phaeocystis pouchetii (Haptophyta) to viral infection. Aquat Microb Ecol 48: 105-112.

[8] Boraas M, Seale D, Boxhorn J (1998) Phagotrophy by a flagellate selects for colonial prey: A possible origin of multicellularity. Evol Ecol 12: 153-164.

[9] Kirk, DL (1997). Volvox: a search for the molecular and genetic origins of multicellularity and cellular differentiation. Cambridge Univ. Press, New York.

[10] Sathe S, Durand PM (2015) Aggregations of Chlamydomonas reinhardtii cells (Chlorophyceae) can be chimeric and depend on life history traits [in preparation].

[11] Marbà N, Duarte CM, Agustí S (2007) Allometric scaling of plant life history. Proc Natl Acad Sci USA 104: 15777-15780.

[12] Finkel ZV, Beardall J, Flynn KJ, Quigg A, Alwyn T, Rees V, et al (2010) Phytoplankton in a changing world: cell size and elemental stoichiometry. J Plankton Res 32: 119-137.

[13] Banse K, Mosher S (1980) Adult body mass and annual production/biomass relationships of field populations. Ecol Monogr 50: 355.

[14] Landry MR (1978) Predatory feeding behavior of a marine copepod, Luhidocera trispinosd. Limnol Oceanogr 23: 1103-1113.

[15] Landry MR (1980) Detection of prey by CaLanus pacificus: Implications of the first antennae. Limnol Oceanogr 25: 545-549.

[16] Dewitt TJ, Sih A, Hucko JA (1999) Trait compensation and cospecialization in a freshwater snail: size, shape and antipredator behaviour. Anim Behav 58: 397-407.

[17] DeLong JP, Okie JG, Moses ME, Sibly RM, Brown JH (2010) Shifts in metabolic scaling, production, and efficiency across major evolutionary transitions of life. Proc Natl Acad Sci USA 107: 12941-12945.

[18] Michod R (2005) On the transfer of fitness from the cell to the multicellular organism. Biol Philos 20: 967-987. 
[19] Wilson AE, Kaul RB, Sarnelle O (2010) Growth rate consequences of coloniality in a harmful phytoplankter. PLoS ONE 5: e8679.

[20] Jorgensen P, Tyers M (2004) How cells coordinate growth and division. Curr Biol 14: 1014-1027.

[21] Prescott DM (1955) Relations between cell growth and cell division. I. Reduced weight, cell volume, protein content, and nuclear volume of amoeba proteus from division to division. Exp Cell Res 9: 328-337.

[22] Mittermayer C, Braun R, Chayka TG, Rusch HP (1966) Polysome patterns and protein synthesis during the mitotic cycle of Physarum polycephalum. Nature 210: 1133-1137.

[23] Banse K (1982) Cell volumes, maximal growth rates of unicellular algae and ciliates, and the role of ciliates in the marine pelagial. Limnol Oceanogr 27: 1059-1071.

[24] Mitchison JM (2005) Single cell studies of the cell cycle and some models. Theor Biol Med Model 2: 4.

[25] Moseley JB, Mayeux A, Paoletti A, Nurse P (2009) A spatial gradient coordinates cell size and mitotic entry in fission yeast. Nature 459: 857-860.

[26] Martin S, Berthelot-Grosjean M (2009) Polar gradients of the DYRK-family kinase Pom1 couple cell length with the cell cycle. Nature 459: 852-856.

[27] Abedin M, King N (2008) The premetazoan ancestry of cadherins. Science 319: 946-948.

[28] Ratcliff WC, Denison RF, Borrello M, Travisano M (2012) Experimental evolution of multicellularity. Proc Natl Acad Sci USA 109: 1595-600

[29] Koufopanou V (1990) Evolution and development in the flagellate green algae (Chlorophyta, Volvocales). Ph.D. thesis, McGill University.

[30] Walker I, Williams RM (1976) The evolution of the cooperative group. Acta Biotheoretica 25: $1-43$.

[31] Tarnita CE, Taubes CH, Nowak MA (2013) Evolutionary construction by staying together and coming together. $J$ Theor Biol 320: 10-22.

[32] Bonner JT (1998) The origins of multicellularity. Integr Biol 1: 27-36.

[33] Maliet O, Shelton D, Michod RE (2015) Origin of group reproduction during the evolutionary transition to multicellularity. PloS. Comp. Biol. [submitted] 
[34] Michod RE, Shelton DE (2014) Group selection and group adaptation during a major evolutionary transition: insights from the evolution of multicellularity in the Volvocine algae. Biol Theory 9: 452-469. 


\section{Figure Legends}

Figure 1: The complete life cycle diagram

Six states can be identified in the model. They are represented by the general notation $\mathrm{N} x \mathrm{~S} y$, where $x$ represents the number of cells in the state $(1=$ single cell, $2=2$-celled colony, and $4=$ 4 -celled colony) and $y$ represents the cell size $(1=1 \mathrm{x}, 2=2 \mathrm{x}$, and $4=4 \mathrm{x})$. States in the same size class are shown with the same filling pattern. Transitions (growth, division, and splitting) are indicated by arrows, and those representing a group property (beginning with an ending in a group state) are shown in red. The rates of growth, division, and splitting are denoted by $g, d$, and $s$, respectively. Mortality is shown as $\mu$ and states in the same size class have the same mortality.

Figure 2: Five life cycles derived from the complete life cycle diagram in Figure 1 with their associated genotypes $(c, C, N)$. The mutation diagram is shown in a box.

Cycles 1 and 2 are binary fission cycles and differ only in size. Each of these two cycles involve two size classes ( $1 \mathrm{x}$ and $2 \mathrm{x}$ in cycle $1 ; 2 \mathrm{x}$ and $4 \mathrm{x}$ in cycle 2 ). A sequence of growth, division, and splitting makes the binary fission cycle. Cycle 3 represents palintomy and involves all three size classes $(1 \mathrm{x}, 2 \mathrm{x}$, and $4 \mathrm{x})$. Cycle 4 (reverse fission) is similar to binary fission except that growth occurs after division and before splitting. Cycle 5 is the cycle with the largest number of transitions. Splitting in cycle 5 occurs only after two rounds of growth and division. The mutation diagram in the box indicates that from cycle $1 / 1^{\prime}$ it takes at least one mutation to go either to cycle 4 or 5 , and at least one more mutation to reach either cycle 2 or 3 . Cycles 1 and 1' are essentially the same cycle produced by different genotypes. Transitions representing a group property (beginning with an ending in a group state) are shown in red.

\section{Figure 3: Regional analysis for life cycle evolution}

Region 1. Cycle 2 reaches a frequency of 1 (Figure 4A) except on the following two boundaries, where cycle 3 reaches a frequency of 1: (i) boundary with region 2, and (ii) boundary with region 3 (only for sufficiently large values of $\mu 2$ and $\mu 4$; Figure 4B). Region 2. Cycle 3 reaches a frequency of 1 (Figure 4C) except on the boundary with region 4 (only for sufficiently small values of $\mu 2$ and $\mu 4$ ), where cycle 4 reaches a frequency of 1 (Figure 4D). Region 3. Cycle 3 reaches a frequency of 1 in this region (Figure 4E) except in the following parts: $(i)$ for sufficiently small values of $\mu 2$, where cycle 4 approaches a frequency of 1 (Figure 4F), (ii) boundary with region 4 , where cycle 4 reaches a frequency of 1 (Figure 4G), (iii) boundary with region 11 , where cycles 1,3 , and 4 coexist for sufficiently large values of $\mu 1$ and $\mu 4$ (Figure $4 \mathrm{H}$ ), and (iv) boundary with region 1 (only for sufficiently small values of $\mu 2$ and $\mu 4$ ), where cycle 2 reaches a frequency of 1 (Figure 4B). Region 4. Cycle 4 reaches a frequency of 1 in this region (Figure 4I) except on the following two boundaries: (i) boundary with region 2 (only for sufficiently large values of $\mu 2$ and $\mu 4$ ), where cycle 3 reaches a frequency of 1 (Figure 4D), and (ii) boundary with region 12, where cycles 1, 4, and 5 coexist (Figure 4J), and cycle 5 may approach a frequency of 1 . Regions 5-8. Cycle 3 reaches a frequency of 1 (Figures 4K-N). Regions 9 and 10. Cycle 3 reaches a frequency of 1 in these regions (Figures 4O and 4Q) except the following parts, where cycle 1 continues to stay at a frequency of 1: $(i)$ boundary between regions 9 and 11 (only for sufficiently small values of $\mu 1$ and $\mu 2$; Figure 4P), and (ii) boundary between regions 10 and 12 (only for sufficiently small values of $\mu 1$ and $\mu 2$; Figure 4R). Region 
11. Cycle 1 maintains a frequency of 1 (Figure 4S) except on the following boundaries: $(i)$ boundary with region 9 (only for sufficiently large values of $\mu 1$ and $\mu 2$ ), where cycle 3 reaches a frequency of 1 (Figure 4P), and (ii) boundary with region 3 , where cycles 1,3 , and 4 coexist (Figure 4H). Cycle 3 becomes dominant and approaches a frequency of 1 as we decrease the values of $\mu 1$ and $\mu 4$. Region 12: Cycle 1 maintains a frequency of 1 (Figure 4T) except on the following boundaries: $(i)$ boundary with region 4 , where cycles 1 , 4, and 5 coexist. Cycle 5 becomes dominant and approaches a frequency of 1 as we decrease the values of $\mu 1$ and $\mu 4$, and (ii) boundary with region 10 (only for sufficiently large values of $\mu 1$ and $\mu 2$ ), where cycle 3 reaches a frequency of 1 . Growth in regions in the top half the diagram accelerates and in the bottom half decelerates with size. Cycles are small fission (cycle 1; red), large fission (cycle 2; green), palintomy (cycle 3; dark blue), reverse fission (cycle 4; brown), and the complex cycle (cycle 5; light blue). The color of each region reflects the dominant cycle in accordance with curve colors in Figures 4 and 5.

\section{Figure 4: Typical examples in different regions and their boundaries}

Simulations were continued until all cycles reach a steady state. The $\mathrm{x}$ and $\mathrm{y}$ axes indicate time and the frequency of each cycle in the population, respectively. (A) Region 1, (B) boundary between regions 1 and 3 (for sufficiently large values of $\mu 2$ and $\mu 4$ ), (C) region 2, (D) boundary between regions 3 and 4 (for sufficiently small values of $\mu 2$ and $\mu 4$ ), (E) region 3, (F) region 3 for sufficiently small values of $\mu 2$, (G) boundary between regions 3 and $4,(\mathbf{H})$ boundary between regions 3 and 11 (for sufficiently large values of $\mu 1$ and $\mu 4$ ), (I) region 4 , (J) boundary between regions and 12, (K) region 5, (L) region 6, (M) region 7, (N) region 8, (O) region 9, (P) boundary between regions 9 and 11 (for sufficiently small values of $\mu 1$ and $\mu 2$ ), (Q) region 10, (R) boundary between regions 10 and 12 (for sufficiently small values of $\mu 1$ and $\mu 2$ ), (S) region 11, (T) region 12. Cycles shown in each panel are small fission (cycle 1), large fission (cycle 2), palintomy (cycle 3 ), reverse fission (cycle 4), and the complex cycle (cycle 5). 


\section{Tables}

Table 1: Summary of the cycles with their genotypes, important features, and examples

\begin{tabular}{|l|c|c|c|l|l|}
\hline \multirow{2}{*}{ Cycle } & \multicolumn{2}{|c|}{ Genotype } & \multirow{2}{*}{ Important features } & Examples \\
\cline { 2 - 6 } & $\boldsymbol{c}$ & $\boldsymbol{C}$ & $\boldsymbol{N}$ & Impoll & $\begin{array}{l}\text { Common among prokaryotes } \\
\text { and small, unicellular eukaryotes }\end{array}$ \\
\hline 1 (small binary) & 2 & 2 & 2 & $\begin{array}{l}\text { A cell grows 2-fold and divides once. The products of } \\
\text { division split. }\end{array}$ & $\begin{array}{l}\text { Common among prokaryotes } \\
\text { and small, unicellular eukaryotes }\end{array}$ \\
\hline 1' (small binary) & 2 & 2 & 4 & $\begin{array}{l}\text { A cell grows 2-fold and divides once. The products of } \\
\text { division split. }\end{array}$ & $\begin{array}{l}\text { Common among prokaryotes } \\
\text { and small, unicellular eukaryotes }\end{array}$ \\
\hline 3 (palintomy) & 4 & 4 & 4 & $\begin{array}{l}\text { A cell grows 4-fold and then undergoes two rounds of } \\
\text { division. The products of the second division split. }\end{array}$ & $\begin{array}{l}\text { Common among walled } \\
\text { chlorophytes }\end{array}$ \\
\hline 4 (reverse fission) & 2 & 4 & 2 & $\begin{array}{l}\text { Growth occurs after division and before splitting, rather } \\
\text { than vice versa, while cells are grouped }\end{array}$ & $\begin{array}{l}\text { Occurs in members of the } \\
\text { Tetrabenaceae }\end{array}$ \\
\hline 5 (complex) & 2 & 4 & 4 & $\begin{array}{l}\text { Growth and division are interspersed, as with binary fission. } \\
\text { Splitting occurs after two rounds of growth and division. }\end{array}$ & \\
\hline
\end{tabular}




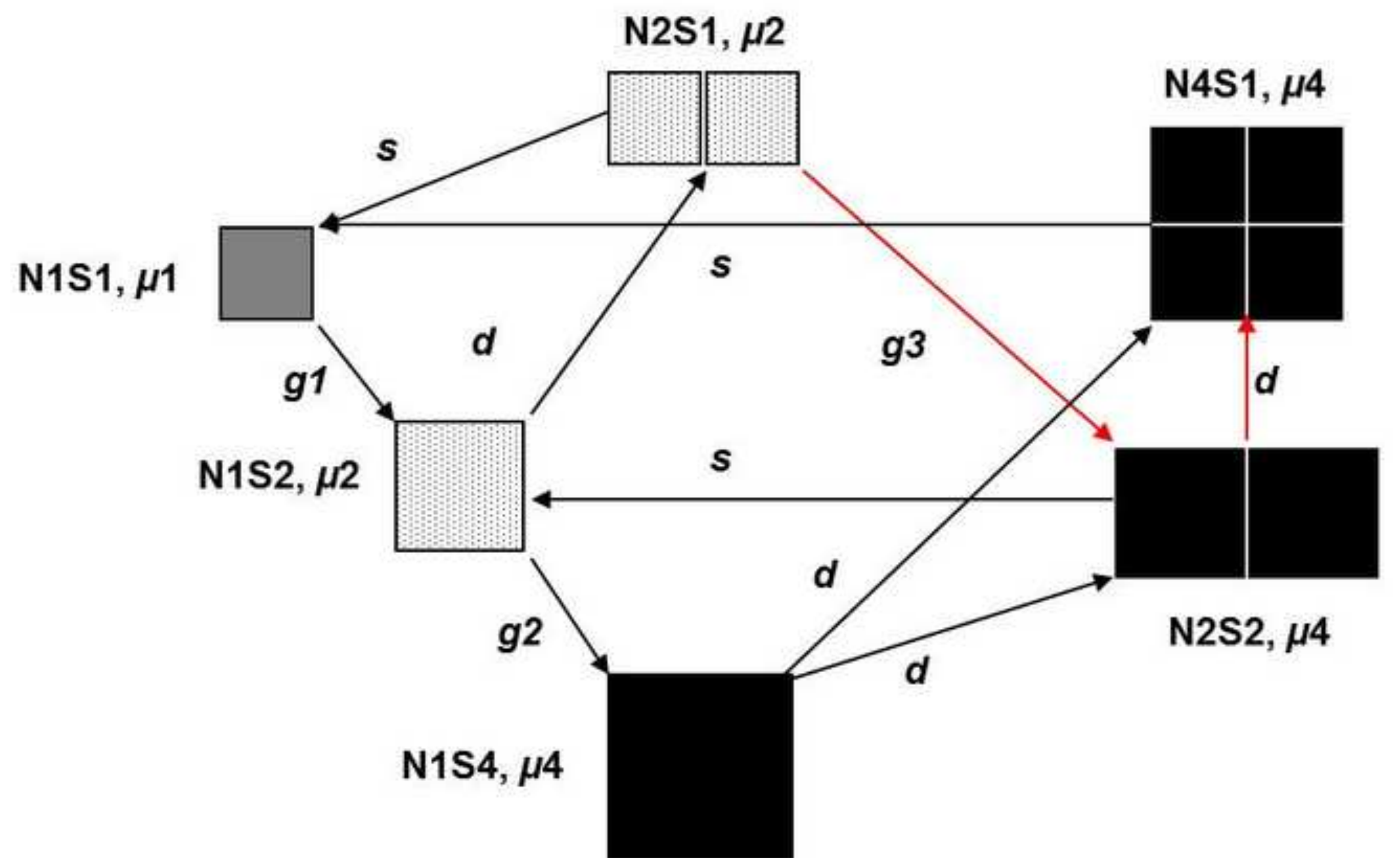




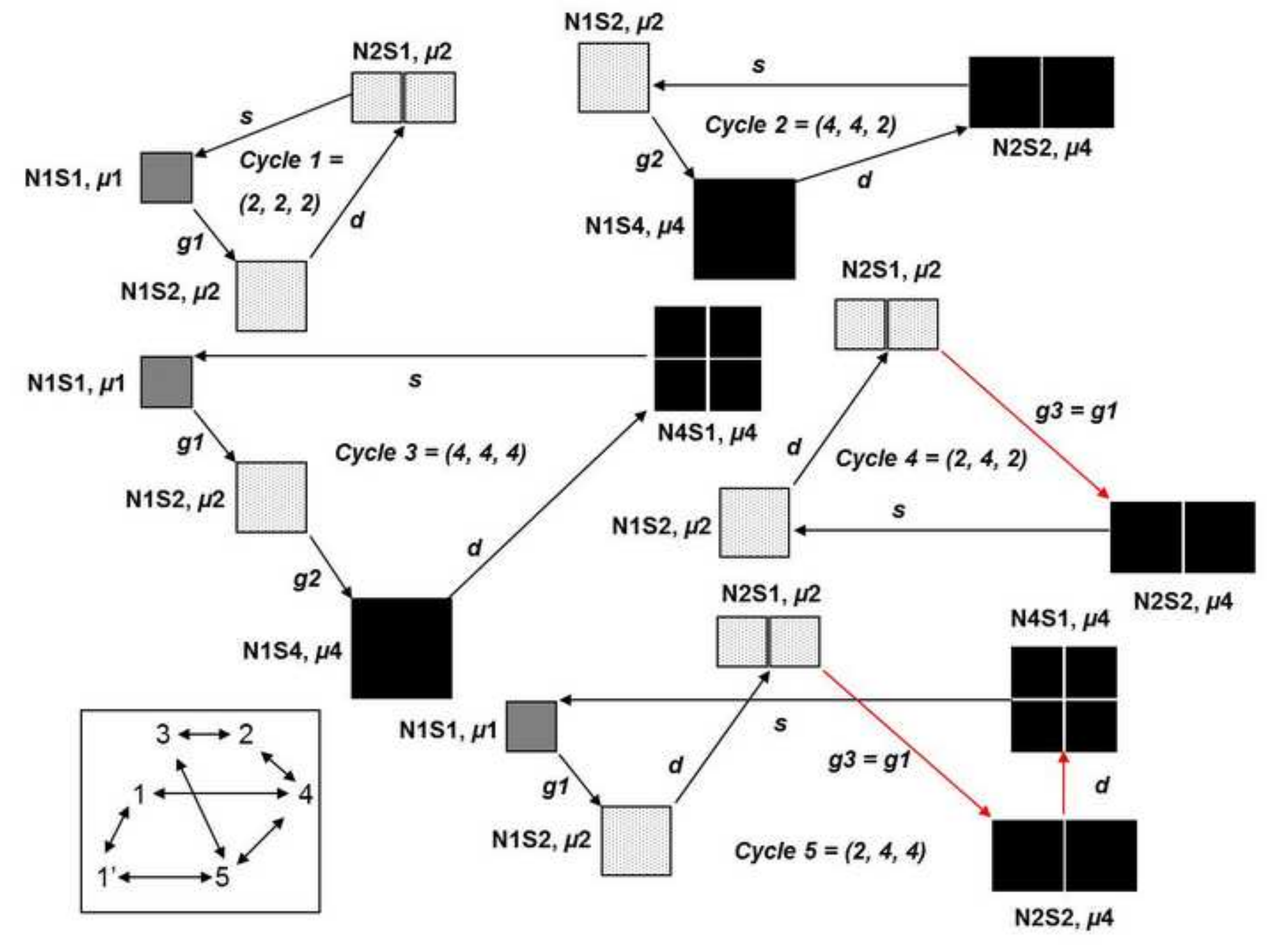




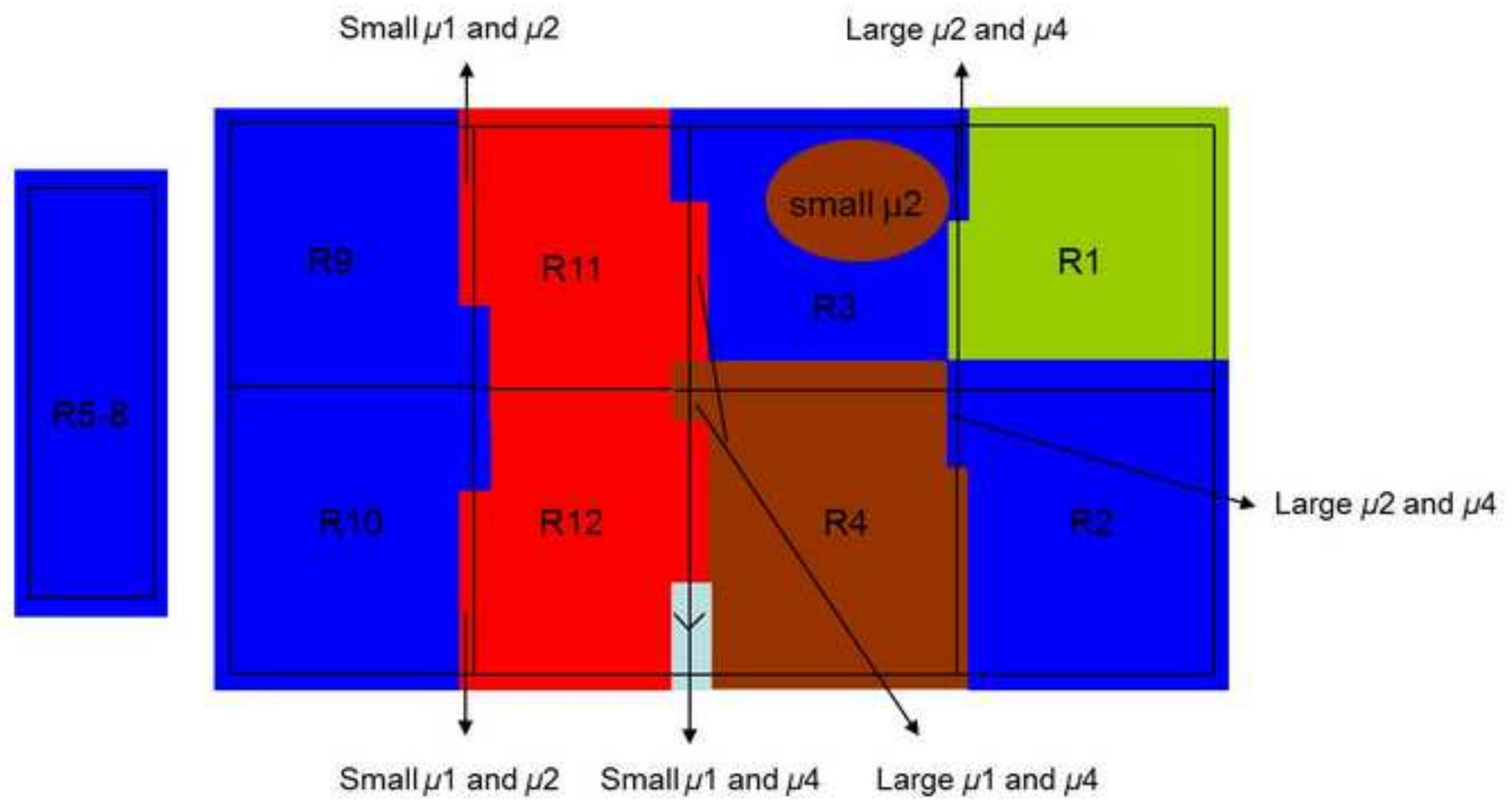



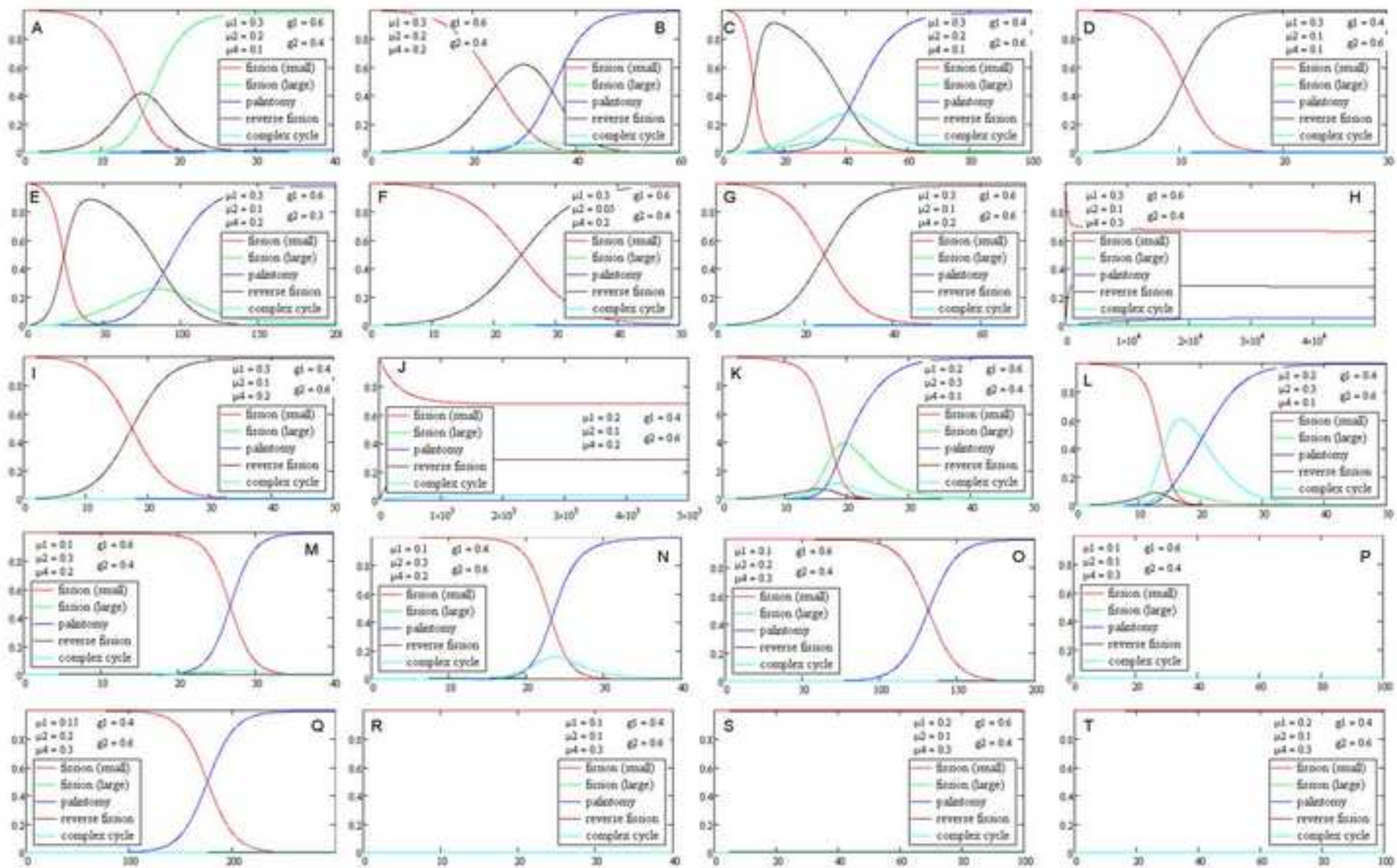